\title{
Protecting Water Quality and Public Health Using a Smart Grid
}

\author{
Ken Thompson ${ }^{1}$, Raja Kadiyala ${ }^{2}$ \\ ${ }^{1}$ Intelligent Water Solutions, CH2M HILL, Englewood, Colorado, USA \\ ${ }^{2}$ Intelligent Water Solutions, CH2M HILL, Oakland, California, USA \\ Email: ken.thompson@ch2m.com,raja@ch2m.com
}

Received 2013

\begin{abstract}
After the attacks on September 11, 2001 and the follow-up risk assessments by utilities across the United States, securing the water distribution system against malevolent attack became a strategic goal for the U.S. Environmental Protection Agency. Following 3 years of development work on a Contamination Warning System (CWS) at the Greater Cincinnati Water Works, four major cities across the United States were selected to enhance the CWS development conducted by the USEPA. One of the major efforts undertaken was to develop a process to seamlessly process "Big Data" sets in real time from different sources (online water quality monitoring, consumer complaints, enhanced security, public health surveillance, and sampling and analysis) and graphically display actionable information for operators to evaluate and respond to appropriately. The most significant finding that arose from the development and implementation of the "dashboard" were the dual benefits observed by all four utilities: the ability to enhance their operations and improve the regulatory compliance of their water distribution systems. Challenge: While most of the utilities had systems in place for SCADA, Work Order Management, Laboratory Management, 311 Call Center Management, Hydraulic Models, Public Health Monitoring, and GIS, these systems were not integrated, resulting in duplicate data entry, which made it difficult to trace back to a "single source of truth." Each one of these data sources can produce a wealth of raw data. For most utilities, very little of this data is being translated into actionable information as utilities cannot overwhelm their staffs with manually processing the mountains of data generated. Instead, utilities prefer to provide their staffs with actionable information that is easily understood and provides the basis for rapid decision-making. Smart grid systems were developed so utilities can essentially find the actionable needle in the haystack of data. Utilities can then focus on rapidly evaluating the new information, compare it known activities occurring in the system, and identify the correct level of response required. Solution: CH2M HILL was engaged to design, implement, integrate, and deploy a unified spatial dashboard/smart grid system. This system included the processes, technology, automation, and governance necessary to link together the disparate systems in real time and fuse these data streams to the GIS. The overall solution mapped the business process involved with the data collection, the information flow requirements, and the system and application requirements. With these fundamentals defined, system integration was implemented to ensure that the individual systems worked together, eliminating need for duplicate data entry and manual processing. The spatial dashboard was developed on top of the integration platform, allowing the underlying component data streams to be visualized in a spatial setting. Result: With the smart grid system in place, the utilities had a straightforward method to determine the true operating conditions of their systems in real time, quickly identify a potential non-compliance problem in the early stages, and improve system security. The smart grid system has freed staff to focus on improving water quality through the automation of many mundane daily tasks. The system also plays an integral role in monitoring and optimizing the utilities' daily operations and has been relied on during recovery operations, such as those in response to recent Superstorm Sandy. CH2M HILL is starting to identify the processes needed to expand the application of the smart grid system to include real-time water demands using AMI/AMR and real-time energy loads from pumping facilities. Once the smart grid system has been expanded to include Quality-Quantity-Energy, CH2M HILL can apply optimization engines to provide utility operations staffs with a true optimization tool for their water systems.
\end{abstract}

Keywords: Smart Grid; On-line Water Quality Monitoring; OWQM; Event Detection System; EDS; TEVA-SPOT

\section{Introduction}

Continuous monitoring of distribution system water quality was rarely conducted prior to the terrorist attacks of September 2001 on the United States. Following those events and the completion and review of risk assessments for all public water systems (PWSs) serving a population 
greater than 3,300, the distribution system was identified as the most vulnerable area of attack.

Homeland Security Presidential Directive 9 required the U.S. Environmental Protection Agency (EPA) to develop a process for utilities to improve the protection of their water distribution systems. In response, distribution system water quality monitoring pilot projects were conducted, which were funded by the EPA Water Security Division Water Security (WS) initiative. [1] As a result, continuous monitoring systems are in operation in Cincinnati, Dallas, New York, Philadelphia, and San Francisco. Independent from the WS initiative program, some PWSs and U.S. government agencies have been developing similar programs. Benefits of these systems include improvement of water treatment processes, increased efficiency of water utility operations, more assured quality of water delivered to consumers, and increased protection of public health.

\section{Benefits of Distribution System Monitoring}

Benefits from continuous distribution system on-line water quality monitoring (OWQM) may be categorized as operational enhancements, regulatory compliance, and contamination warning.

Operational enhancements include continuous indication of water quality in the distribution system beyond that which is possible through routine regulatory sampling. Early indication may be provided of unusually low residual chlorine levels, impending nitrification (elevated ammonia), turbidity excursions caused by main breaks, and other unusual water quality changes. This monitoring is achieved through measurement of several water quality parameters with which water utilities are already familiar (e.g., chlorine residual) and other parameters that are relatively new to this application (e.g., total organic carbon).

Regulatory compliance benefits of OWQM include improving the ability to maintain chlorine residual as part of the Total Coliform Rule (TCR) [2] and maintaining proper $\mathrm{pH}$ control to avoid potential violations of the Lead and Copper rule [3].

Warning of intentional or unintentional contamination in the distribution system is somewhat more complex. Specialized analyzers are available, including gas chromatographs that may detect specific contaminants and toxicity monitors of many types that can provide a general warning of contamination. Due to the large number of potential contaminants, rather than attempting to specifically identify a contaminant, it is more practical to monitor for an indication of contamination through changes in many of the same water quality parameters, or surrogates, that are used for operational benefit monitoring.
Beyond monitoring for operational and contamination purposes, utilities should consider OWQM as part of the Distribution System Optimization program of the Partnership for Safe Water [4]. The Partnership is a voluntary effort between EPA, the American Water Works Association (AWWA), several other drinking water organizations, and more than 200 water utilities throughout the United States. The goal of the Partnership is to provide a new measure of safety to millions of Americans by implementing prevention programs where legislation or regulation does not exist to do so. The preventative measures are focused on optimizing treatment plant performance and distribution system operation.

\section{Selection of Water Quality Parameters}

A water utility that is embarking on design of distribution monitoring must first decide which water quality parameters to monitor. Parameters that are typically included in OWQM systems include:

- Total organic carbon (TOC)

- Residual chlorine

- Conductivity

- $\mathrm{pH}$

- Turbidity

These parameters are of interest to utilities from a distribution system operational and regulatory perspective and provide critical information including:

- TOC - Elevated turbidity excursions can be associated with a breakthrough at the water treatment plant (WTP) or scouring and release of biofilm within the distribution system.

- Residual chlorine - A sudden loss in residual could promote biofilm growth and potential violation of the TCR.

- Conductivity - This measurement provides an easy method for identifying mixing or different water sources, which can have a significant impact on many industrial operations.

- $\mathrm{pH}-\mathrm{pH}$ is controlled for disinfection and corrosion control. The formation of some disinfection byproducts is $\mathrm{pH}$ dependent.

- Turbidity - This parameter provides warning of a system disruption created by a surge or reversal in flow that scours the pipeline. This could be caused by a pipeline break, hydrant knockover, or other problems that will impact chlorine residual and customer satisfaction.

Utilities that use chloramines for disinfection also measure for ammonia, nitrates, and dissolved organic carbon (DOC) to provide early warning of nitrification in the distribution system. The first water quality indicator of nitrification will be the increase of ammonia, which will occur before nitrites and nitrates begin to increase.

The myriad potential contaminants have been classified among twelve categories by EPA [5]. Laboratory 
testing has concluded that three of these water quality parameters-TOC, residual chlorine, and conductivity-respond to the presence of contaminants from ten of the twelve categories, so along with operational monitoring, broad contaminant coverage is also provided with a minimum of instrumentation. The relative change in quality of water with either chlorine or chloramines has been investigated by EPA and the pilot cities to serve as a general guidance for evaluating a water quality anomaly.

Most OWQM systems include monitoring for absorbance of ultra-violet (UV) light. UV analyzers, which operate by measuring absorption at the single 254-nanometer $(\mathrm{nm})$ wavelength, are generically referred to as UV-254 analyzers. Instruments that operate through analysis of a broad spectrum from 200 to $720 \mathrm{~nm}$ are referred to as spectral analyzers.

UV absorbance has been shown to be strongly correlated to TOC content [6]. UV-254 analyzers measure absorbance at the 254-nm wavelength as this is the radiation emitted by a common mercury-based UV source lamp. The absorbance of UV light by TOC or other contaminants contained within the water sample is reported as a percent of the uninhibited lamp intensity, ranging from 0 to 100 percent.

Spectral analyzers utilize a xenon lamp to produce a light source across UV and visible light wavelengths from 200 to $720 \mathrm{~nm}$. Measurement of absorbance at 254 discrete wavelengths across this range enables construction of an absorbance, or spectral, curve (Figure 1). Due to the substantially greater information provided by spectral analysis, the broadband spectrum enables measurement of TOC based on calculation of the numerous $\mathrm{UV} /$ visible light wavelengths that are associated with this parameter. Similarly, turbidity is also calculated based on analysis of numerous wavelengths. Subtraction of the turbidity component enables derived measurement of nitrate and DOC. Other parameters, not typically included in OWQM analysis, also may be derived from broad spectral absorbance of UV and visible light.

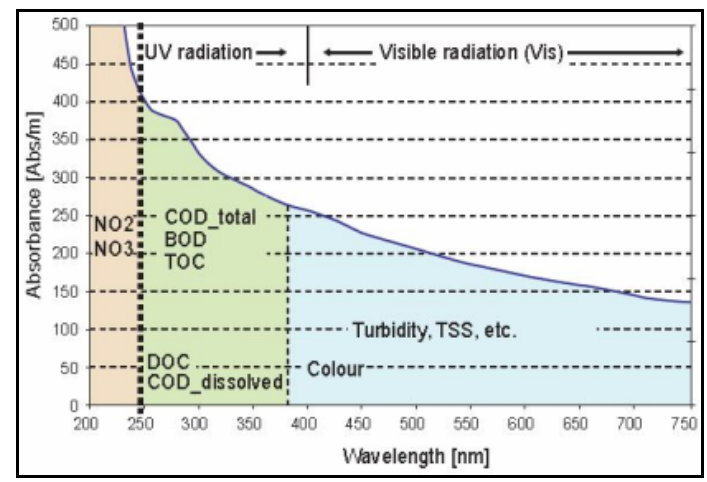

Figure 1. Ultraviolet/Visible Absorbance spectrum enables calculation and derivation of numerous water quality parameters.
Not all sources of TOC are revealed by UV/visible light absorbance, but a large enough percentage are detected that these technologies are generally accepted for the OWQM application. This limitation of correlated or spectral indication of TOC must be considered when selecting OWQM parameters.

\section{Selection of Water Quality Analyzers}

Once water quality parameters to be used for OWQM monitoring are selected, specific instruments to be used for this purpose must be selected. All utilities are under pressure to minimize capital and operating costs, so this consideration factors into analyzer selection. Addition of OWQM monitoring must be done without unnecessarily adding to the existing responsibilities of utility technicians, also impacting the selection of OWQM analyzers. One way to do this is to select sensors and analyzers that are reliable and inexpensive to operate, and require only infrequent direct attention or maintenance.

\subsection{Chlorine Analyzers}

The two most common methods for on-line chlorine analysis are amperometric and colorimetric detection. The colorimetric method requires use of chemical reagents to produce a reaction, which is measured and used to quantify chlorine content. Reagent reservoir levels must be regularly monitored and refilled by maintenance personnel. Additionally, reagents used for colorimetric tests have been found to degrade in environments that exceed 105 degrees, significantly affecting the quality of the analysis.

Amperometric sensors measure changes in electric current or potential and operate without use of chemical reagents. For monitoring residual chlorine by OWQM systems, these sensors reduce maintenance costs and activities, as well as operational risks from depletion of reagent reservoirs between service visits. Therefore amperometric technologies are most frequently used.

\subsection{TOC Analyzers}

Traditional TOC analyzers involve multiple electrochemical reactions for operation. Phosphoric acid is used for $\mathrm{pH}$ reduction and inorganic carbon removal, followed by oxidation of organic carbon to $\mathrm{CO}_{2}$ by sodium or ammonium persulfate and heat or UV light. At least one manufacturer uses boron-doped electrodes to generate oxidation radicals in place of the persulfate solution.

$\mathrm{CO}_{2}$ is directly detected by a non-dispersive infrared (NDIR) detector, or converted to carbonic acid and measured by conductance. Operation requires periodic replenishment of acid and, if used, persulfate reagents. The UV lamp, when used, also requires replacement. 
Prefiltration of the analyzer sample stream is frequently required to prevent plugging of the micro-tubing that is included in the analyzer's internal construction. In some water that includes a substantial level of inorganic carbon, additional filters to remove this carbon component must be included at the inlet to the analyzer. These filtering systems are generally available from the analyzer manufacturer, but constitute a maintenance activity and additional cost that varies depending upon the particular nature of the sample stream.

Mechanically, TOC analyzers are highly complex and require substantial technical training and experience to ensure proper operation over extended periods [7]. While analyzers that are mechanically less intricate are now commercially available, they remain complex in operation.

\subsection{UV Analyzers}

When $\mathrm{UV}_{254}$ analysis is used for an OWQM system, the absorbance measurement alone may be used as a general indication of water quality. Due to the potential difficulties involved with operating and maintaining TOC analyzers, UV analyzers are sometimes used to provide a TOC measurement or indication, depending on the particular technology selected.

Several factors must be considered when selecting a UV absorbance analyzer for use in OWQM systems.

Some $\mathrm{UV}_{254}$ analyzers apply a correlation coefficient to the $254 \mathrm{~nm}$ absorbance reading to generate a TOC measurement. However, the accuracy of the correlation is dependent upon the stability of the correlation. For systems where the source water is subject to change, the correlation may change, and without adjustment to the programmed coefficient, the TOC measurement may be inaccurate.

TOC correlation to $\mathrm{UV}_{254}$ absorbance is also impacted by the turbidity of the sample stream. Some $U_{254}$ analyzers include automated turbidity compensation, while others do not.

Spectral analyzers calculate TOC and turbidity measurement from the UV and visible light absorption measurements that make up the spectral curve. Turbidity readings from these analyzers are applied to the TOC calculation to achieve a turbidity-compensated TOC measurement. These analyzers can also derive nitrate, DOC and other water quality parameters. Specific water contaminants (e.g., ricin [8]) can be calculated based on their specific absorbance spectrum, similar to the method used for TOC determination.

EPA studies have found that UV/visible light absorbance-based TOC readings will not detect all TOC compounds, but they do detect a large enough portion of potential TOC contaminants that these instruments are valid for contamination monitoring [9]. Similarly, TOC read- ings by these instruments are suitable for indicating distribution system water quality because drinking water TOC is typically made up of humic and fulvic acids, which are very accurately detected by UV absorption.

Absorbance-based UV and TOC measurements have the potential to be affected by deposition of mineral content on optical surfaces. Highly dependent on the water being tested, mineral deposits on lenses will impact absorbance readings. Optically based analyzers frequently include automated cleaning systems ranging from periodic flushing with various solutions to mechanical wipers or brushes, or continuous operation of ultrasonic wave generators. These have been found to be of varying effectiveness.

Some UV-based optical analyzers include automated compensation for variation of the UV lamp output over time. This function is essential as lamp output is known to decrease over time, requiring periodic lamp replacement.

\subsection{Ammonia Analyzers}

Ammonia sensors may be reagent or non-reagent based. For OWQM systems, reagentless technologies that use ion-selective electrodes are preferred to minimize maintenance activities and costs.

The ammonia measurement should include $\mathrm{pH}$ compensation, which may be integral to the ammonia sensor or a separate $\mathrm{pH}$ sensor with signal input to the ammonia analyzer. The $\mathrm{pH}$ signal can also be separately used for the OWQM pH measurement. Ammonia sensors may also include potassium compensation because elevated potassium levels will interfere with low-level ammonia measurements, such as those close to the sensor detection limit where OWQM systems typically operate. The potassium signal is not an OWQM parameter and is not separately reported by the OWQM system.

\subsection{Conductivity, pH, Turbidity Analyzers}

These sensors operate using standard, proven electrochemical and optical technologies that water utilities have deployed in WTPs and other facilities for many years. Specific analyzers to be used in an OWQM station should be those that the user has found to be reliable in service and to provide accurate readings with minimal maintenance requirements. For these parameters, use of a utility's standard sensors is usually acceptable.

\section{Prioritization of Installation Locations}

Selection of installation locations for OWQM stations involves important considerations to reduce cost and provide an environment conducive to long-term and successful operations.

Monitoring stations are typically installed at the dis- 
charge of each WTP or at wholesale connection interties to indicate baseline conditions entering the distribution system for comparison with downstream measurements. These stations also indicate results of changes to the treatment process and warn of conditions in the treatment process that may otherwise go undetected. Some OWQM process measurements may already be made at the WTP, and these existing measurements can be used for OWQM purposes. When adding other OWQM parameters that are not already monitored at the WTP, some utilities use installation of OWQM stations as an opportunity to upgrade older instruments or to convert to reagentless technologies to reduce labor and maintenance costs.

OWQM stations are frequently installed at the discharge of distribution system reservoirs and chlorine boosting pump stations. Measurement may be upstream of chlorine addition to provide a measure of the quality of the water in the reservoir and that further upstream. The OWQM station could alternately be installed downstream of chlorine addition at a booster station to provide a baseline measurement for comparison to OWQM stations further downstream.

OWQM stations can also be installed at critical nodes in the distribution system, and different approaches may be taken in selecting these locations. Distribution system managers generally have a good understanding of the operation of the piping network and can often identify the nodes of interest based on experience. A more scientific approach to OWQM siting is frequently conducted through the use of the Threat Ensemble Vulnerability Analysis and Sensor Placement Optimization Tool (TEVASPOT) software [10]. This analytical package, developed by EPA, Sandia Laboratories and others, analyzes a distribution system network and identifies critical nodes that will represent water quality impacting the largest number of consumers. TEVA-SPOT analysis results often validate the operational understanding expressed by distribution system managers, but also frequently identify nodes otherwise not understood to be critical or recommend subtle location changes compared to the managers' recommendations.

The analysis enables prioritization of a selected number of OWQM stations to meet the budget available for a project and identify stations to be added as a monitoring system expands over a period of years.

\section{Requirements for Data Communication and Analysis}

Several products are available for analysis of OWQM data and alarming of unusual conditions. The most common software event detection systems (EDSs) are commercially available through s::can, Whitewater, and Hach. Also available is the Sandia National Laboratories freeware system Canary, which was developed as part of the
WS initiative. Each EDS has strengths and weaknesses associated with its performance under distribution system operations. Additional information can be obtained through the EPA Water Security Division.

OWQM alarms are generally based on more than simple alarm setpoints for parameter measurements. Typically the alarms or alerts are associated with pattern alarms, where multiple parameters change in a manner that is atypical of their normal relationship. As an example, if TOC increased, it would also be expected that DOC would increase in a proportional manner. When a utility implements enhanced coagulation, the TOC-toDOC relationship changes, generating an alert at the water quality monitoring stations. Broadband UV/visible systems also produce a spectral alarm that is initiated if the normal spectral fingerprint displays an unusual shift.

OWQM data are collected more frequently than typical Supervisory Control and Data Acquisition (SCADA) monitoring data, and therefore OWQM data usually cannot be communicated over traditional low-bandwidth SCADA networks. Additionally, spectral data cannot be communicated over the typical SCADA data collection network, so separate communication pathways must be established. Many utilities, therefore, include all OWQM data on the alternate path and keep monitoring and maintenance of this information separate from operational SCADA data, although there is no requirement to maintain this separation. If $\mathrm{T}-1$ or optical connections to the central monitoring facility are available, these pathways may be used, but typically the OWQM measurements are still transmitted as a separate data stream from SCADA parameters.

Water quality analysis is conducted locally at the OWQM station, and measured values and alarms are communicated to a central historian and display. Typically a longterm database is used for storage and retrieval of data and a short-term cache for short-term (30-day) trending.

For OWQM stations at water utility locations, the data may be communicated over a virtual private network (VPN) set up on the existing utility network, if available. For locations that do not have access to the utility's network, data are frequently communicated over commercial digital radio or cable service connections.

\section{Fabricated On-line Water Quality Monitoring Stations}

Installed OWQM stations take several forms, depending on the parameters and analyzers selected for use. Outdoor installations are generally fabricated in enclosed cabinets for protection and security. Because water flows inside the cabinet with an open drain, ventilation of the cabinet is required to dissipate moisture that may accumulate. In hot southern climates, temperatures inside the enclosed cabinets are also of concern, so ventilation is 
also necessary and shade from direct solar illumination is recommended. High internal temperatures can also impact the stability of chemical reagents, so reagentless analyzers are also preferred.

Many of these issues may be avoided by installing OWQM stations indoors. For secure indoor locations, such as those owned and controlled by the utility, the station may be configured as a wall-mount panel or open frame system. Heat and moisture are usually no longer an issue, but the potential for tampering or vandalism of equipment may be more of a concern. Indoor locations that are not owned by the utility, such as those at fire departments, police stations, hospitals or other host facilities, should be configured as enclosed cabinets that will still require ventilation to remove humidity. Indoor locations avoid the OWQM environmental impacts of excessive heat or cold, and also make it easier for utility technicians to conduct routine calibration and service. However, indoor installation at any facility requires that access be available on a short-notice basis to retrieve automatically collected water samples in the event potential contamination is detected. Continuous access is a priority for locating OWQM stations that hold contamination monitoring as a mission-critical consideration. For those focused only on operational benefits, quick access is less of a siting priority.

\section{Operational Benefits}

The most important aspect of OWQM systems are the benefits of assured and improved water quality provided to the consumer. Such monitoring may provide recommendations for adjustment of the water treatment process and feedback of the results of treatment process changes. OWQM stations can provide early warning of water main breaks, low or high chlorine conditions, nitrification, and other conditions and thereby not only improve operations, but can also save considerable costs to the utility.

When the EDS identifies an anomaly in a data stream, an alert is sent to the centralized distribution system monitoring dashboard, which allows the operator to spatially correlate information from other data streams (consumer complaints, enhanced security alarms, public health alerts) in real time. Operations staff can rapidly and independently query each alert to evaluate trends and relate the anomalies to explainable events that may be occurring in the water distribution system (e.g., a pipeline break). During this operational evaluation, it is possible that the cause of the alerts cannot be explained by known activities. When this occurs, the utility can proceed with a tiered response, i.e., a Consequence Management Plan, that becomes increasingly aggressive as more information related to a potential contamination event is received.
The ability of rapidly converting very large data streams into actionable information and providing the consolidated alert information on a user-friendly dashboard significantly decreases a utility's response time. The ability to identify trends and nuances in the data supports operational benefits not previously available. Examples of collected data associated with distribution system events are presented below.

Figure 2 is a plot of spectral absorbance that indicated a peak characteristic of iron oxide. Plots of the data in five-day increments showed the size of the peak was progressively increasing. This trend was ultimately identified as accumulating deposition of iron on the analyzer optics, caused by aggressive water unexpectedly leaching from ductile iron pipe. Identifying and addressing the problem early saved the utility an estimated \$20 million in early pipe replacement costs.

In Figure 3, DOC and TOC plots were used to optimize granular activated carbon (GAC) filter performance. In this case the utility developed correlations between total trihalomethane (TTHM) production and effluent DOC for use in determining when to change the GAC and maintain system compliance. In this example the utility reduced the annual replacement costs by $\$ 100,000$ at each WTP.

Figure 4 illustrates how OWQM data can be used to track water age. In this case, nitrate profiles were compared over time to determine travel time between sites. The data were validated through the utility's calibrated model and similar readings from other distribution system locations were used to verify the hydraulic model.

Figure 5 shows an example of how spectral absorbance changes indicated failure of treatment plant controls early enough for the problem to be resolved before major damage occurred. In this case, the failure allowed spent brine solution to flow into the distribution system reservoir. The immediate result was a spectral change associated with the highly colored brine solution blocking the

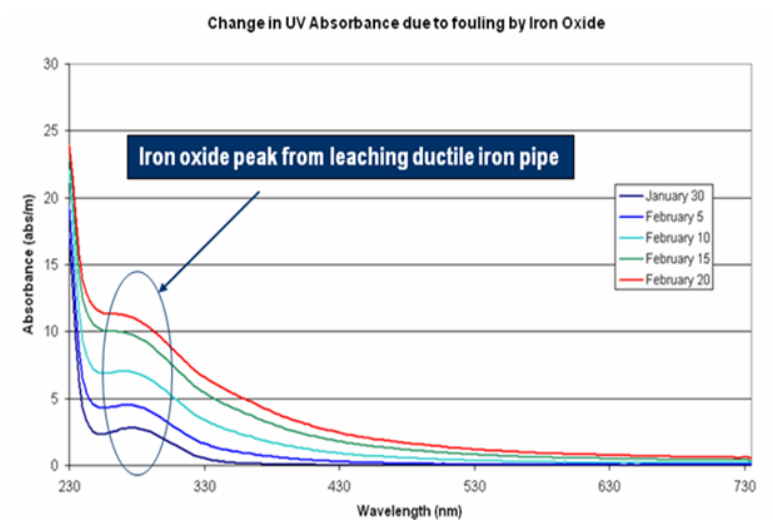

Figure 2. On-line water quality monitoring ultraviolet absorbance spectrum enables identification of distribution system accelerated corrosion. 


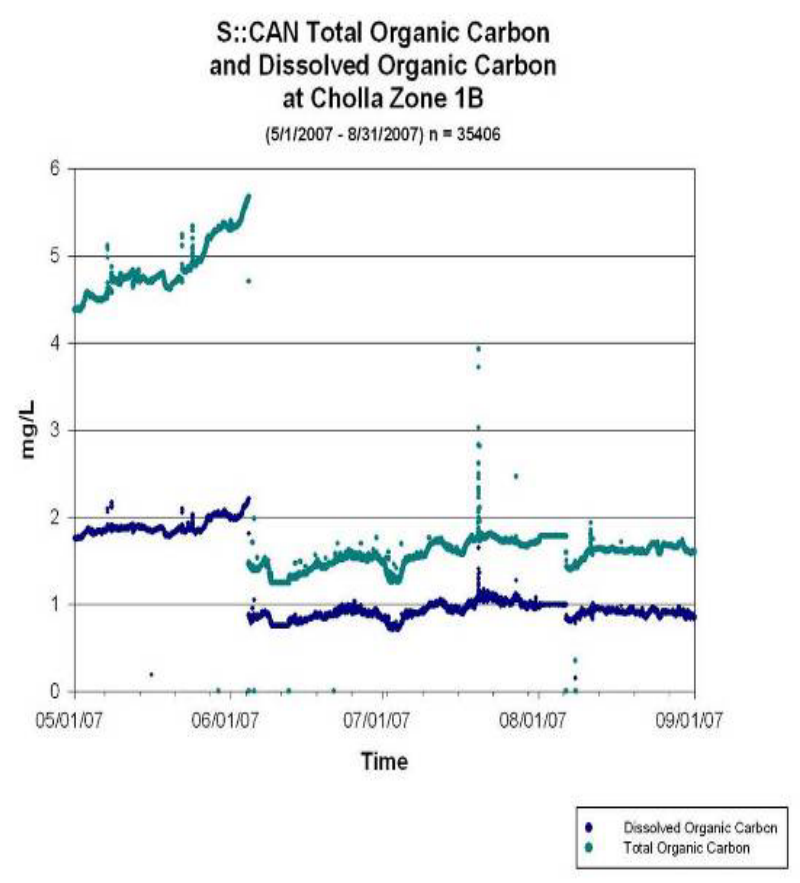

Figure 3. On-line water quality monitoring dissolved organic carbon and total organic carbon plots used to optimize treatment plant maintenance activities.

UV transmittance and creating a spectral alarm and notification. The graph on the left indicates normal absorbance (blue trace at bottom, behind and coincident with green trace). Over a period of several minutes, the absorbance increases, with maximum disruption indicated by the orange and purple marks indicating high absorbance of 30 to 50 percent for three minutes. Thirteen minutes after the start of the event, the condition was resolved, the brine solution had passed the monitoring station, and the absorbance spectrum returned to normal (green trace at bottom, coincident with the pre-event absorbance).

The right graph shows conditions the next day at monitoring station in the distribution system. The con-

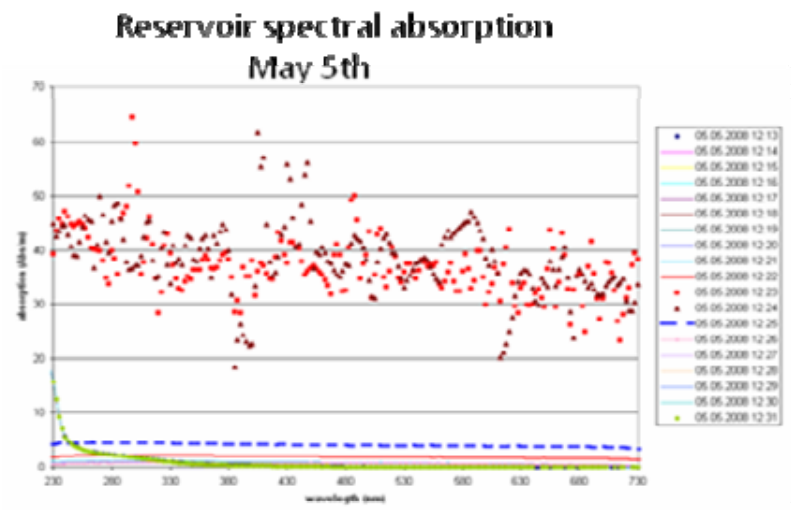

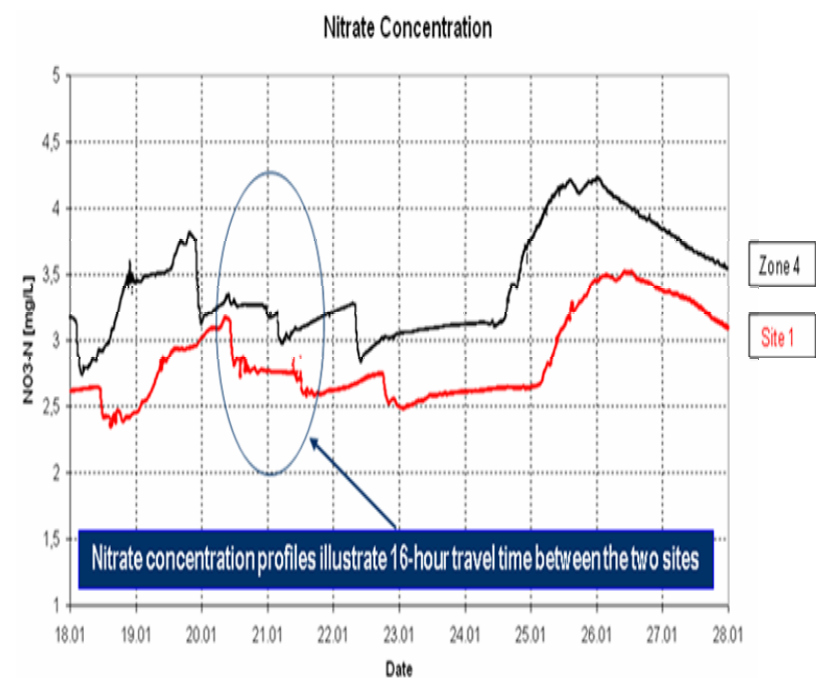

Figure 4. On-line water quality monitoring data enables comparison of nitrate profiles and hydraulic model verification.

taminant is shown to be considerably diluted, with the maximum absorption reduced to 30 to 40 percent and spread over seven minutes. The event is seen to take a total of four hours to pass the station from start of the event to return to normal water quality.

A military base that uses water from a local utility often had reports of water quality problems that could not be readily attributed to a known cause. Monitoring of the water inlet to the base identified changes in the water supply as shown in Figure 6. The water provider occasionally changed the source of the water and where it entered the distribution system. The result was heavy scouring in the pipe, directly impacting water quality on the base. Once the problem was understood, the base was able to work with the water provider to inform them of the impact associated with flow reversals in the distribution system. The water provider also implemented a flushing program to reduce the sediment that had accumulated in the distribution system pipelines.

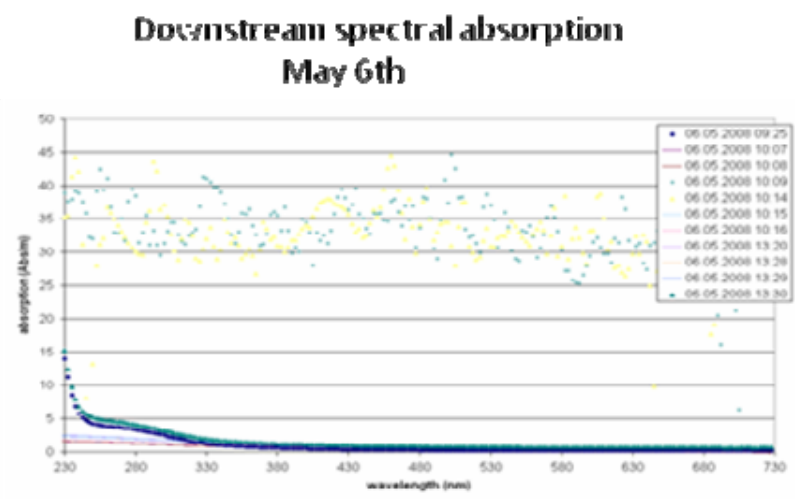

Figure 5. On-line water quality monitoring-identified changes in spectral characteristics indicate equipment failure. 

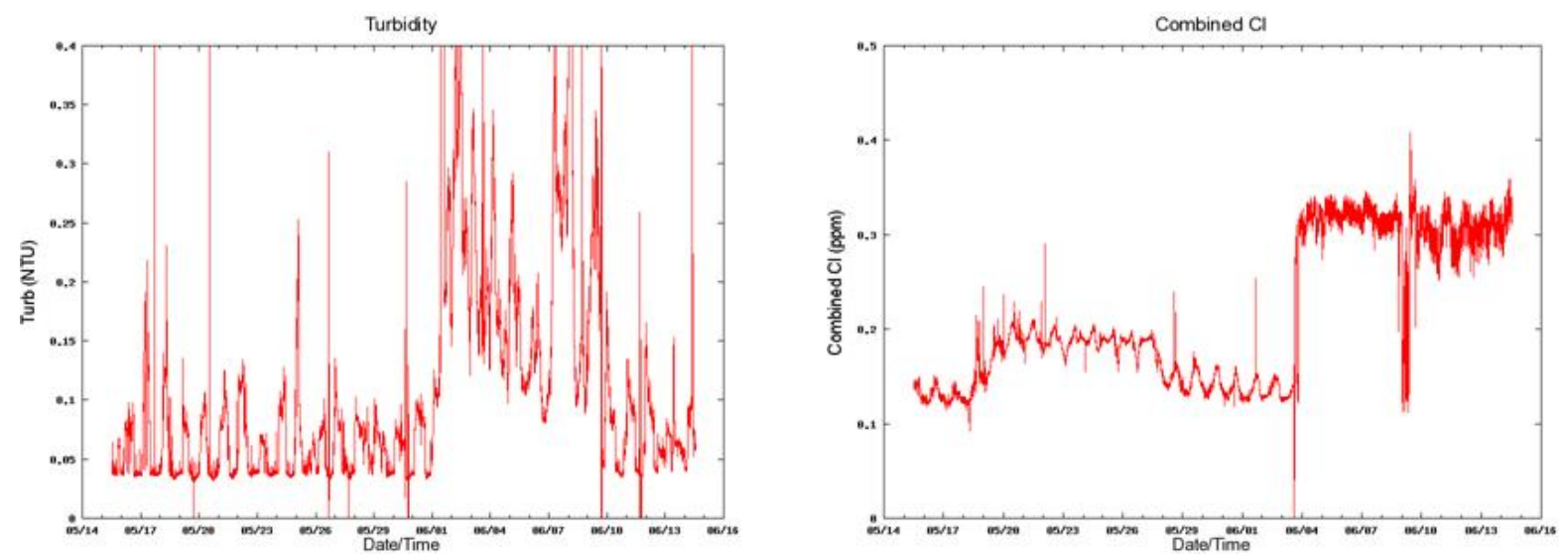

Figure 6. On-line water quality monitoring system identifies changes in source water as the cause of water quality problems.

\section{Operational Benefits}

Installation and operation of OWQM stations at WTP and reservoir outlets, and at strategic locations throughout the distribution system provides an understanding of water delivery conditions that may have been previously unknown or incompletely understood. These systems enable correction of problems and improvement in delivered water quality, provide added protection of public health, and produce a substantial savings in operational and maintenance/replacement costs. A mix of well known and new technology sensors and analyzers may be used to generate the substantial amount of data required for a complete picture of distribution system operation. The data can be displayed in an optimized manner on a central dashboard as well as through mobile technologies to support water utility operations.

\section{REFERENCES}

[1] CH2M HILL, "Contamination Warning System Demonstration Pilot Project: Implementation and Assessment," 2013.

[2] EPA. (n.d.) "The Effectiveness of Disinfectant Residuals in the Distribution System," Washington, DC: US EPA Office of Water, Office of Ground Water and Drinking Water.

http://www.epa.gov/ogwdw/disinfection/tcr/pdfs/issuepap er_effectiveness.pdf
[3] EPA, "Revised Guidance Manual for Selecting Lead and Copper Control Strategies," Washington, DC: US EPA Office of Water, Office of Ground Water and Drinking Water, 2003.

[4] AWWA. (n.d.) Partnership for Safe Water Program Fact Sheet: Treatment Plant and Distribution System Optimization.

http://www.awwa.org/Portals/0/files/resources/water\%20 utiity\%20management/partnership\%20safe \%20water/files /FactSheetRevSept20102.pdf

[5] EPA, "Water Sentinel Online Water Quality Monitoring as an Indicator of Drinking Water Contamination," Washington, DC: US EPA Water Security Division, 2005.

[6] J. Dilling and K. Kaiser, "Estimation of the hydrophobic fraction of dissolved organic matter in water samples using UV photometry," Water Resourse, Vol. 36, No. 20, 2002, pp. 5037-5044. doi:10.1016/S0043-1354(02)00365-2

[7] J. Hall and J. Szabo, "Distribution System Water Quality Monitoring: Sensor Technology Evaluation Methodology and Results," Cincinnati, OH: EPA, 2009.

[8] J. van den Broeke, "Spectral RICIN Measurement - Detection Limits," s: can Messtechnik, GmbH, 2009.

[9] EPA, "Water Security Initiative: Interim Guidance on Planning for Contamination Warning System Deployment," Cincinnati, OH: EPA Water Security Division, 2007.

[10] J. E. Berry, TEVA-SPOT Toolkit and User's Manual. Washington, DC: EPA, 2008. 